\title{
Dynamic Hip Screw Compared to Condylar Blade Plate in the Treatment of Unstable Fragility Intertrochanteric Fractures
}

\author{
CK Yong, MS Ortho, CN Tan*, MS Ortho, R Penafort**, MS Ortho, DA Singh, MS Ortho, \\ MV Varaprasad***, FRCS (Edin) \\ Orthopaedic \& Trauma Surgery, Prince Court Medical Centre, Kuala Lumpur, Malaysia \\ *Department of Orthopaedic Surgery, Putrajaya Hospital, Putrajaya, Malaysia \\ **Department of Orthopaedic and Trauma Surgery, KPJ Damansara Specialist Centre, Petaling Jaya, Malaysia \\ ${ }^{* * *}$ Department of Orthopaedic Surgery, University Malaya Medical Centre, Kuala Lumpur, Malaysia
}

\begin{abstract}
Dynamic hip screw (DHS) fixation is considered standard treatment for most intertrochanteric fractures. However, excessive sliding at the fracture site and medialisation of femoral shaft may lead in fixation failure. In contrast, fixedangled $95^{\circ}$ condylar blade plate (CBP) has no effective dynamic capacity and causes little bone loss compared to DHS. We compared the outcome of 57consecutive unstable intertrochanteric fragility fractures treated with these two fixation methods. CBP instrumentation is more difficult requiring longer incision, operating time and higher surgeonreported operative difficulty. The six month post-operative mortality rate is $16 \%$. Post-operative Harris hip scores were comparable between the two methods. Limb length shortening more than $20 \mathrm{~mm}$ was 6-fold more common with DHS. In elderly patients with unstable intertrochanteric fragility fractures, fixed angled condylar blade plate appears to be a better choice than dynamic hip screws for preventing fixation failures.
\end{abstract}

Key Words:

Unstable Intertrochanteric Fractures; Extramedullary fixation; Low Fragility; Dynamic Hip Screw; $95^{\circ}$ Condylar Blade Plate

\section{INTRODUCTION}

Fragility fractures can be caused by a fall from a standing height or less. Osteoporosis which leads to bone fragility is considered the major contributing factor to fragility fractures and is directly linked to risk of hip fracture. Every year there are 250,000 hip fractures in the United States, and it is predicted that this number will double in the next 40 years ${ }^{1}$. A similar increase is expected in Asia ${ }^{2}$. In general, as the population ages, the incidence and cost for treating these injuries is going to increase dramatically ${ }^{3,4}$. Hip fractures are associated with high morbidity and mortality, a rate of up to $20 \%$ can be expected in the year following the injury ${ }^{5}$. The majority of those who survive are disabled with only $25 \%$ able to resume normal activities ${ }^{6}$.

About half of the hip fragility fractures are intertrochanteric fractures. Dynamic hip screw (DHS) fixation has been considered the gold standard for treatment of stable intertrochanteric fractures ${ }^{7,8}$. DHS allows controlled collapse of the fracture followed by progressive stabilization. However, there are divergent opinions about the fixation of unstable intertrochanteric fractures in the elderly. Authors reported high failure rates (range: $3 \%$ to $26 \%$ ) for DHS fixation in unstable intertrochanteric fractures. Failure usually occurs due to loss of fixation of the lag screw with resultant varus angulations and medial collapse at the fracture site; plate pull-off from the shaft; implant disassembly; or fatigue failure in cases of delayed union $9,10,11,12$. Orthopaedists have questioned whether the fixation should be dynamic by default allowing fracture compression and union at the cost of reduced femoral neck length and medialisation of the femur shaft. Or should rigid fixation applied to restore the pre-fracture anatomy to achieve immediate stability for early mobilization in a race towards fracture union before implant fatigue?

There are limitations as to how much of dynamic fracture compression is desirable. DHS fixation permits fracture compression along the femoral neck that leads to femoral neck shortening; there is also an inherent tendency towards medialisation of the femoral shaft ${ }^{13}$. Jacobs et al found that the average length of lag screw sliding was $5.3 \mathrm{~mm}$ in stable fractures, and $15.7 \mathrm{~mm}$ in unstable fractures. Excessive sliding (more than $15 \mathrm{~mm}$ ) correlates to higher prevalence of fixation failure ${ }^{4}$, and is also associated with increased post- 
operative pain and decreased post-operative mobility ${ }^{14}$. Parker found that if medialisation of more than one-third of the femoral shaft diameter occurs at the fracture site, there is a seven-fold increased risk of fixation failure ${ }^{15}$.

A fixed-angled $95^{\circ}$ condylar blade plate (CBP) can be used in these difficult unstable fractures as well as in revision fixation for failed intertrochanteric fractures ${ }^{16}$. This technique results in improved resistance to rotation of the proximal fragment, as it has no effective dynamic capacity. This is due to the fact that, on loading, the hip joint reaction force is $159^{\circ}$ towards the vertical plane. CBP does not allow the proximal fragment to slide laterally thus avoiding the undesirable medialisation of the shaft.

We compared these two extramedullary fixation devices in the treatment of unstable intertrochanteric fractures.

\section{MATERIALS AND METHODS}

Consecutive patients admitted in 2004 with unstable intertrochanteric fractures were prospectively randomized (by drawing lots from sealed envelopes) into two study groups. The study was approved by the hospital ethics committee. Consent was also obtained from patients or, in cases where they were confused, from their caretakers. Inclusion criteria were: 55 years of age or older with low traumatic intertrochanteric fracture classified as AO/OTA Type 31 A2.2 and A2.3 and Kyle Type III and Type IV. They were fractures with comminution, loss of postero-medial calcar, subtrochanteric extension, and reverse oblique fracture pattern ${ }^{17}$. Two-part fractures were excluded since they are considered stable fractures. Patients with fractures associated with polytrauma, pathological fractures, and previous surgery on the ipsilateral hip or femur were excluded.

There were 61 patients with 63 hip fractures treated during the study period. 57 hips are included in the resutls: 31 treated with DHS (Dynamic Hip Screw, Synthes-Stratec, Oberdorf, Switzerland) and 26 treated with $\mathrm{CBP}\left(95^{\circ}\right.$ condylar blade plate; Synthes-Stratec, Oberdorf, Switzerland). Six patients were excluded as five were unfit for surgery and therefore treated conservatively, while one was transferred to other centre. Surgery was performed within four days in all patients. Best possible alignment is achieved either by closed manipulation under fluoroscopy control or open reduction. Standard method of instrumentation and fixation were performed under fluoroscopy control. The modified Harris Hip Score without assessing the hip motion was used to determine pre-fracture status, and the Harris Hip Score (HSS) was used for postoperative 3rd and 6th month functional assessment. Plain radiographs were taken before and one day post-operatively, followed by repeated radiographs at 1st, 3rd and 6th month postoperatively. Post-operative rehabilitation was standardized in both groups with emphasis on early gain of protected partial weight-bearing using a walking frame before discharge.

Categorical variables were analyzed using chi-square tests of association (ambulatory status). Continuous variables were assessed by One-way ANOVA test or Student's t-test (Harris Hip Score, operating time, blood loss and Visual Analogue Score of operating difficulty). The level of significance was set at $p<0.05$.

\section{RESULTS}

The subjects in both groups were similar in terms of demographic and premorbid functional status (Table I). With CBP the incision was typically longer and blood loss was also more but these differences were not statistically significant. Skin to skin operative time was significantly longer using CBP, though the clinical relevance of a mean of extra 20 minutes is unclear. When coupled with a significantly higher surgeon-reported Visual Analogue Score (VAS) in operating difficulty, a longer operative time rightly reflects the difficult CBP instrumentation (Table II).

Within 3 months following surgery, $10 \%$ of the patients passed away from medical complications unrelated to their surgery, with another $5 \%$ in the following 3 months, contributing to a $16 \%$ mortality 6 month after surgery (Table III). Harris Hip Scores 3 months and 6 months after surgery were comparable between the 2 groups. Twenty-one per cent of surviving patients at did not regain the ability to walk when assessed 6 months after surgery. There was no correlation between the ability to walk and limb length shortening or choice of implant. Despite early surgical intervention, the $16 \% 6$ month mortality rate and the high rate of failure to return to ambulatory status were attributed to the systemic nature of osteoporosis in the elderly.

\section{Complications}

There was no recognised deep vein thrombosis, pulmonary embolism, or deep infection. There were 3 fixation failures in each group due to suboptimal surgical fixation (Table IV). In the DHS group, poor reduction with medialisation of femoral shaft by $25 \%$, a short lag screw and/or maximal initial sliding leaving no room for further fracture impaction, eventually led to superior cut out in 3 patients (Figure 1). This resulted in two patients remaining bedridden, and in the third patient with no acetabulum involvement, revision fixation was performed 6 months later. Shortening (leg length difference of more than $20 \mathrm{~mm}$, clinically reported and radiologically measured) was significantly more common in the DHS group.

In the CBP group, the failure to primarily restore neck-shaft angle, as evidenced by the post-operative pelvic anteroposterior film, led to one case of excessive varus, nonunion 
and subsequent implant fatigue at the 4th postoperative month. There was one nonunion leading to superior cut out in the CBP group (Figure 2). The overall failure rate, when limb length shortening and fixation failures were considered, was lower in group CBP and approaching statistical significance with $\mathrm{p}$ value at 0.51 . Medialisation of femoral shaft was not compared, as it was not possible with the fixedangle CBP.

Table I: Demographic and premorbid functional evaluation and fracture pattern

\begin{tabular}{|lcc|}
\hline & \multicolumn{2}{c|}{ Method of fixation } \\
& DHS $(\mathbf{n = 3 1})$ & $17: 9$ \\
\hline Women: Men & $28: 3$ & $78(58-96)$ \\
Age (years) : mean (range) & $78(55-100)$ & 77.66 \\
QCT scan (mg/cm3) : mean BMD & 74.72 & $77(52-91)$ \\
Modified pre fracture Harris HIP score; mean (range) & $80(52-91)$ & 17 \\
Walking ability, prefracture & & 9 \\
$\quad$ No aids & 21 & 0 \\
$\quad$ Aids & 10 & 18 \\
$\quad$ Non walker & 0 & 8 \\
Fracture Type (AO/OTA type 31) & & 20 \\
$\quad$ A2.2 & 11 & \\
A2.3
\end{tabular}

Table II: Per operative parameters

\begin{tabular}{|lccc|}
\hline & \multicolumn{2}{c|}{ Method of fixation } & P value \\
\hline Operating time (min) : mean (range) & DHS ( $\mathrm{n}=9)$ & CBD $(\mathrm{n}=10)$ & $0.001^{\mathrm{b}}$ \\
Operating blood loss (litre) : mean (range) & $74(50-120)$ & $94(45-135)$ & $0.27^{\mathrm{b}}$ \\
Operating difficulty ${ }^{\text {a }}$ mean (range) & $0.19(0.05-0.80)$ & $0.24(0.05-0.80)$ & $0.018^{\mathrm{b}}$ \\
\hline
\end{tabular}

a Visual Analogue Score: 1 (easy) - 10 (difficult)

${ }^{b}$ One-way ANOVA test

Table III: Post-operative mortality rate

\begin{tabular}{|lccc|}
\hline & DHS $(\mathbf{n}=\mathbf{3 1})$ & CBP $(\mathbf{n}=\mathbf{2 6})$ & Percentage (\%) \\
\hline Deceased & 1 & 5 & 10.5 \\
$<3$ months & 1 & 2 & 5.3 \\
$3-6$ months & & & \\
\hline
\end{tabular}

Table IV: Complication and failures of the procedures

\begin{tabular}{|c|c|c|c|}
\hline & DHS $(n=31)$ & $C B P(n=26)$ & p value \\
\hline \multicolumn{4}{|l|}{ Fixation complication } \\
\hline Greater trochanter fracture & 2 & 3 & \\
\hline Poor reduction & 1 & 1 & \\
\hline \multicolumn{4}{|l|}{ General complication } \\
\hline Superficial infection & 1 & 1 & \\
\hline Deep infection & 0 & 0 & \\
\hline Limb length shortening $>20 \mathrm{~mm}$ & 6 & 1 & $0.045^{\mathrm{a}}$ \\
\hline \multicolumn{4}{|l|}{ Fixation failures } \\
\hline Superior cut out & 3 & 1 & $0.391^{\mathrm{a}}$ \\
\hline Breakage/loosening & 0 & 1 & \\
\hline Nonunion & 0 & 1 & \\
\hline Revision & 1 & 0 & \\
\hline \multicolumn{4}{|l|}{ Failure rate } \\
\hline Limb length shortening $>20 \mathrm{~mm}$ \& Fixation failures & $9 / 31(29 \%)$ & $4 / 26(15 \%)$ & $0.051^{\mathrm{a}}$ \\
\hline
\end{tabular}

Table V: Quantitative Computed Tomography (QCT) scan of selected patients

\begin{tabular}{|lcc|}
\hline & DHS $(n=9)$ & CBP $(n=10)$ \\
\hline QCT scan (mg/cm3) : mean (range) & 74.72 & 77.66 \\
\hline
\end{tabular}




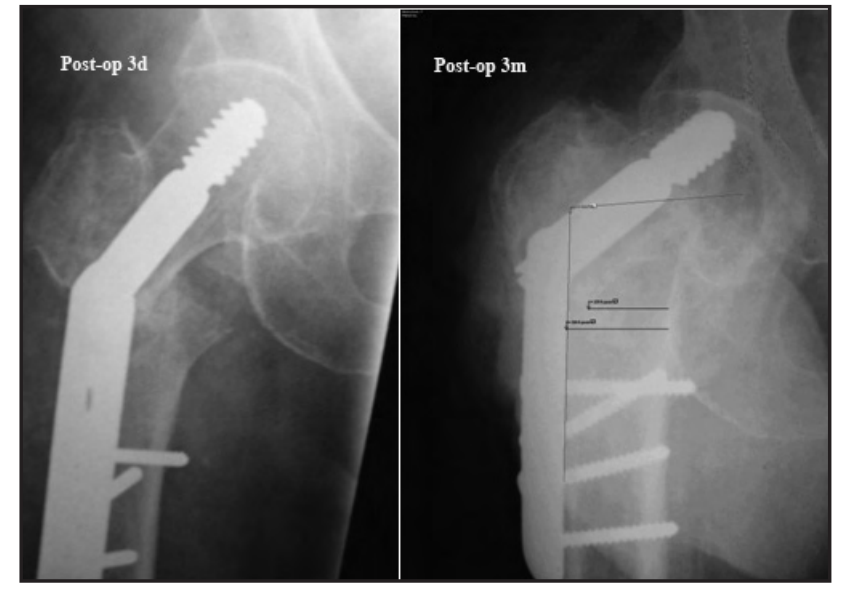

Fig. 1: Superior cut out and shaft medialization following fixation with dynamic hip screw fixation.

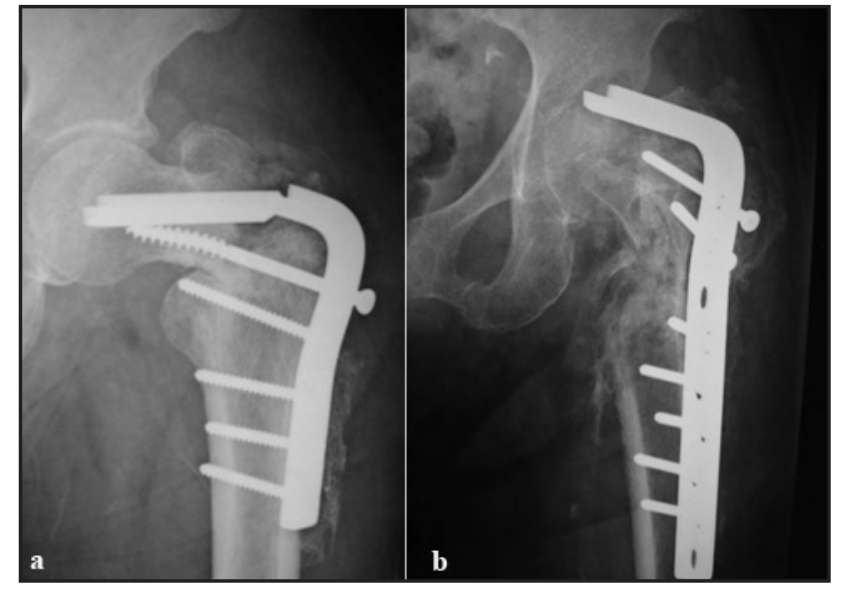

Fig. 2: Failure patterns following treatment with $95^{\circ}$ fixed angle condylar blade plate fixation in 2 patients.

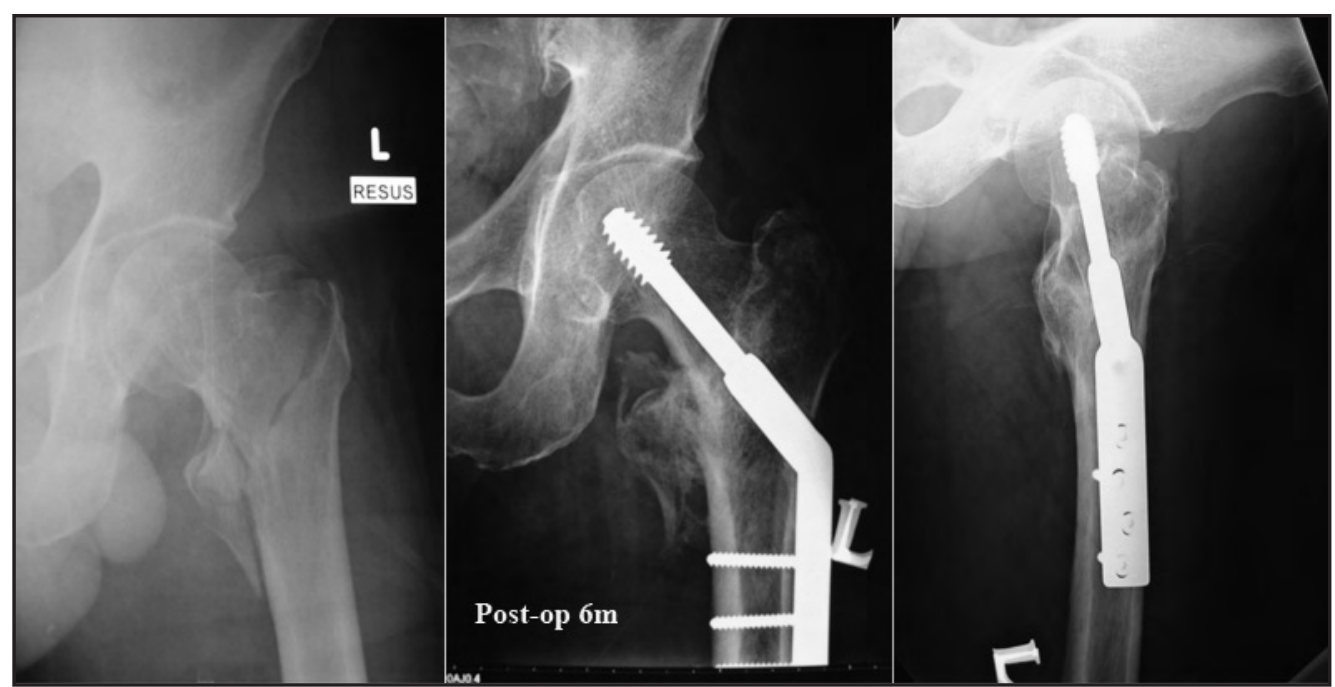

Fig. 3: A 3-part intertrochanteric fracture with no postero-medial calcar support united following dynamic hip screw fixation.

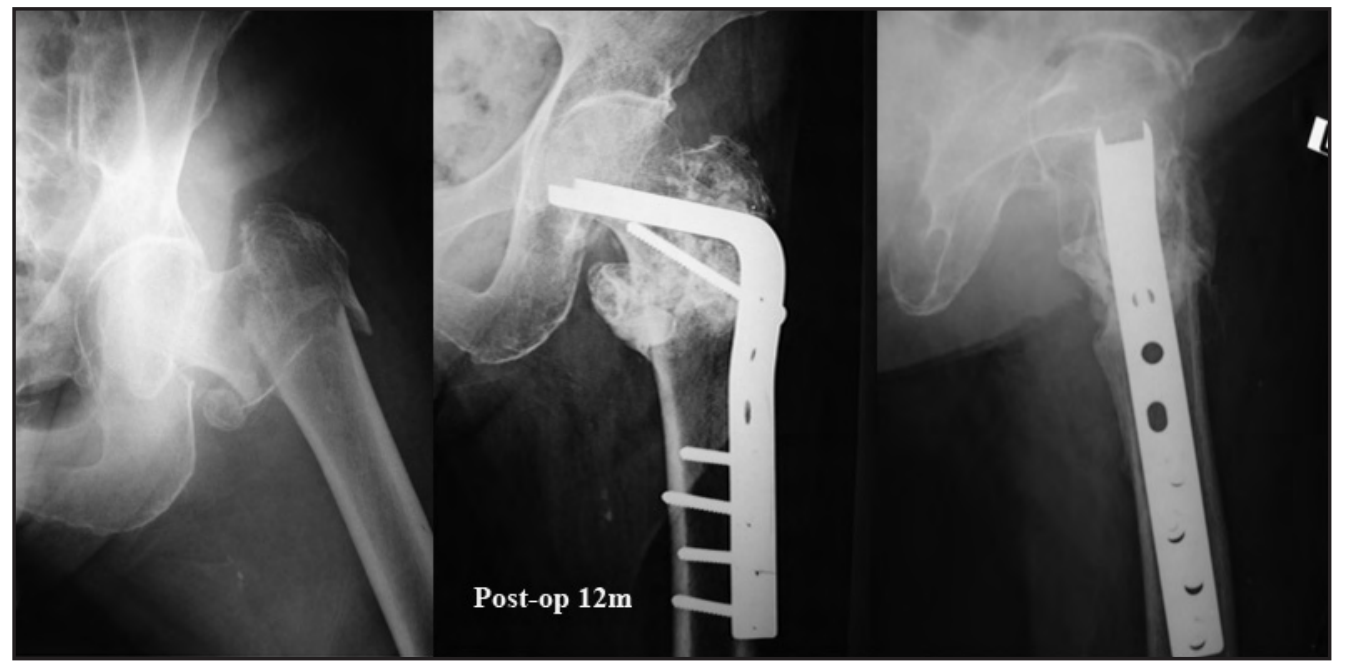

Fig. 4: Florid callus formation following $95^{\circ}$ fixed angle condylar blade plate fixation for a comminuted intertrochanteric fracture using bridge plating technique. 


\section{DISCUSSION}

For the fixation of stable intertrochanteric fractures, there exists a plethora of choices of implants, whereas the ideal implant remains elusive for unstable intertrochanteric fractures. Unstable intertrochanteric fractures especially those with a posterior medial defect are more prone to complications. Loss of posterior medial support leads to increase hip screw telescoping and limb shortening. Further, these defects also lead to increased load transfer to the tip of the dynamic hip screw, thereby increasing superior cut out. Loss of fixation of the lag screw in an osteoporotic head, with resultant varus angulation and medial collapse at the fracture site can also occur ${ }^{18}$. Independent of the type of implant used, patients with unstable trochanteric hip fractures and osteoporotic bone are at the highest risk for implant failure ${ }^{19}$.

Kaufer determined that the strength of the implant-fracture composite is based on 5 variables: bone quality, fracture geometry, fracture reduction, placement of implant, and implant used ${ }^{20}$. A CT scan of an intertrochanteric fracture may reveal poor density of trabeculation and even emptiness within the head. Maximum bone density is likely found at the site where the compressive and tensile trabeculae coalesce, in the centre-centre region of the femoral head ${ }^{21}$. An ideal implant should engage this region while taking as little remaining strong bone as feasible. The $\mathrm{CBP}$ at $95^{\circ}$ to the femur shaft is wedged below the densest trabeculae in the centre-centre region of the femoral head. There is little bone loss in preparing the sitting bed for CBP especially as compared to DHS that requires bone coring.

Of concern is unstable fracture geometry that include fractures with increased comminution, loss of posteromedial calcar, subtrochanteric extension, and reverse oblique fractures. Anatomic reduction of femoral neck length, axial length, and neck-shaft angle must be achieved before implantation. In addition to the degree of osteoporosis, the rate of superior cut out is strongly correlated to surgical technique. Baumgaertner et al demonstrated that enhanced surgeon awareness of short tip-apex distance (TAD) decreases the risk of fixation failure ${ }^{22}$.

Choice of implant should not be routine but customised according to fracture characteristics. Unstable fractures with osteoporosis are reported to have failure rate of more than
$50 \%{ }^{23}$. In such cases DHS may still yield good outcome (Figure 3) but DHS should not be automatically the first choice for treatment. Our results indicate that unstable intertrochanteric fractures, treated with fixed-angled $95^{\circ}$ CBP have similar outcomes and near statistically significant lower failure rate compared to DHS. This fixed-angle device acts as a bridge plate across the fracture site, and therefore may be of value in severe osteoporotic bones as well as in comminution where dynamic fixation may lead to excessive interfragmentary compression. Conversely, the two failure patterns of CBP observed in present study, namely implant breakage and nonunion, may be due to lack of intefragmentary compression. For optimal application of the bridging plate principle across fracture comminution, the authors recommend the use of a longer 7 or 9-hole side plate CBP with 4 distal screws and 1 proximal lag screw for the femoral head. Subsequent cases conducted using this technique consistently led to fracture union (Figure 4).

The present study has several limitations. Though both devices analyzed in this study are extramedullary using similar surgical exposure, the more difficult CBP instrumentation may be subject to performance bias; hence a larger studied population is warranted. Secondly, only some of the patients underwent a Quantitative Computed Tomography (QCT) scan. Among the patients who were scanned, there was no difference in bone mineral density detected between the two groups, but inclusion of all patients may have reveal subtle differences (Table V). Thirdly, although body mass index was not studied, it may be an important factor in early stability of fracture-implant construct and subsequent union rate. Of course, follow-up longer than 6 months would have been optimal. Lastly as the focus was on extramedullary fixation, the present study did not include any intramedullary device options for these fractures.

\section{CONCLUSION}

In the treatment of fragility fractures, it is important to recognise unstable fracture geometry and customise the choice of implant according to the fracture characteristics. In elderly patients with unstable fragility intertrochanteric fractures, fixed angled condylar blade plate appears to be a better choice than dynamic hip screws with a lower rate of fixation failure. 


\section{REFERENCES}

1. Cummings SR, Rubin SM, Black D. The future of hip fractures in the United States: Numbers, costs, and potential effects of post menopausal estrogen. Clin Orthop Relat Res 1990; 252: 163-6.

2. Lau EMC, Lee JK, Suriwongpaisal P. The Incidence of hip fracture in four Asian countries: The Asian Osteoporosis Study (AOS). Osteoporos Int 2001; 12: 239-43.

3. Jacob RR, McClain O, Armstrong HJ. Internal fixation of intertrochanteric hip fractures: A clinical and biomechanical study. Clin Orthop Relat Res 1980; 146: 62-70.

4. Steinberg GG, Desai SS, Kornwitz NA, Sullivan TJ. The intertrochanteric fracture: A retrospective analysis. Orthop 1988; 11: 265-73.

5. Clayer MT, Bauze RJ. Morbidity and mortality following fractures of the femoral neck and trochanteric region: Analysis of risk factors. J Trauma. 1989; 29(12): 1673-8.

6. Jensen JS, Bagger J. Long term social prognosis after hip fractures. Acta Orthop Scand 1982; 53(1): 97-101.

7. Clawson DK. Trochanteric fractures treated by the sliding screw plate fixation method. J Trauma 1964; 4: 737-52.

8. Kyle RF, Gustilo RB, Premer RF. Analysis of six hundred and twenty-two intertrochanteric hip fractures. J Bone Joint Surg 1979; 61-A, 216-21.

9. Chang CW, Lai KA and Yang CY. Failure of compression hip screw: Analysis of the failure mechanism. J Musculoskeletal Research 2002; 6; 2; 101-6.

10. Leung KS, So WS, Shen WY, Hui PW. Gamma nails and dynamic hip screws for peritrochanteric fractures. A randomised prospective study in elderly patients. J Bone Joint Surg 1992; 74-B: 345-51.

11. Barrios C, Walheim G, Brostrom LA, Olsson E, Stark A. Walking ability after internal fixation of trochanteric hip fractures with Ender Nails or Sliding Screw Plate: A comparative study of gait. Clin Orthop Relat Res 1993; 294: 187-92.

12. Simpson AHRW, Varty K, Dodd CAF. Sliding hip screws: modes of failure. Injury 1989; 20: 227-31.

13. Ceder L, Lunsjö K, Olsson O, Stigsson L, Hauggaard A, Kummer FJ. Can controlled biaxial dynamization lead to improved treatment of complex hip fractures? Orthop Trans 1996; 20: 162-3.

14. Jacob RR, McClain O, Armstrong HJ. Internal fixation of intertrochanteric hip fractures: A clinical and biomechanical study. Clin Ortho Relat Res 1980; 146: 62-70.

15. Parker MJ. Trochanteric hip fractures. Fixation failure commoner with femoral medialization, a comparison of 101 cases. Acta Orthop Scand 1996; 67(4): 329-32.

16. Arthur L, Malkani, Ninad K. Revision surgery for failed intertrochanteric fractures. Techniques in Orthopaedics 2003, 17(4): 443-7.

17. Kyle RF. Current treatment of difficult intertrochanteric fractures. Program and abstracts of the 15th Annual Vail Orthopaedic Symposium; January 18-21, 2001; Vail, Colorado.

18. Laros GS, Moore JF. Complications of fixation in intertrochanteric fractures. Clin Orthop Relat Res 1974; 101: 110-19.

19. Barrios C, Brostrom LA, Stark A, Walheim G. Healing complications after internal fixation of trochanteric hip fractures: The prognostic value of osteoporosis. J Orthop Trauma 1993; 7(5): 438-42.

20. Kaufer H. Mechanics of the treatment of hip injuries. Clin Orthop Relat Res 1980; 146: 53-61.

21. Singh M, Nagrath AR, Maini PS. Changes in trabecular pattern of the upper end of the femur as an index of osteoporosis. $J$ Bone Joint Sur. 1970; 52-A: 457-67.

22. Baumgaertner MR, Solberg BD. Awareness of tip-apex distance reduces failure of fixation of trochanteric fractures of the hip. $J$ Bone Joint Surg 1997, 79-B: 969-71.

23. Kim WY, Han CH, Park JI, Kim JY. Failure of intertrochanteric fracture fixation with a dynamic hip screw in relation to preoperative fracture stability and osteoporosis. Int Ortho 2001; 25: 360-2. 